\title{
Evaluation of Changes in the Ganglionic Cell Inner Plexiform Layer and Macular Retinal Nerve Fibre Layer in Patients Receiving Hydroxychloroquine
}

Mouna Al Saad ( $\nabla$ eye_maas@yahoo.com )

The University of Jordan. School of Medicine https://orcid.org/0000-0002-2189-5259

Amin Shehadeh

Hamad Medical Corporation

Mohammed A. Abu Ameerh

The University of Jordan School of Medicine

Jehad Meqbil

The University of Jordan School of Medicine

\section{Mohammad Qablawi}

The University of Jordan School of Medicine

\section{Saif Al Deen AlRyalat}

The University of Jordan School of Medicine

Khaled Elubous

The University of Jordan School of Medicine

\section{Research article}

Keywords: Hydroxychloroquine, ganglionic cell- inner plexiform layer, macular retinal nerve fiber layer, spectral-domain optical coherence tomography

Posted Date: October 1st, 2020

DOI: https://doi.org/10.21203/rs.3.rs-72241/v1

License: (a) (1) This work is licensed under a Creative Commons Attribution 4.0 International License. Read Full License 


\section{Abstract}

Backgrounds: To evaluate changes in the thickness of ganglionic cell-inner plexiform layer and macular retinal nerve fiber layer using ocular coherence tomographyin patients exposed to hydroxychloroquine .

Methods: This was a retrospective, cross-sectional study of patients on hydroxychloroquine therapy. Ocular coherence tomography images showing of ganglionic cell-inner plexiform cell layer and macular retinal nerve fiber layer thickness were obtained and compared to those of the control groups. The relationship between the thickness of of ganglionic cell-inner plexiform and macular retinal nerve fibre layer and the duration and cumulative dose of hydroxychloroquine was evaluated.

Results: In all, 219 patients were included in this study; the mean age was $43.38( \pm 17.39)$ years. The study group comprised 100 (20 male and 80 female) patients, with a mean age of 45.28 ( \pm 12.24 ) years; the control group had 119 patients (44 males and 75 females), with a mean age of $41.79( \pm 20.67)$ years, with no significant difference in age between the groups $(p=0.123)$. There was a significant difference in mean of ganglionic cell-inner plexiform thickness between the study and control groups $(85.6+/-8 \mu \mathrm{m})$ vs. $(88.6+/-6 \mu \mathrm{m})(\mathrm{p}=0.006)$, with a mean difference of 0.31 ( $95 \%$ confidence interval). The average RNFL thickness was similar in the study and control groups, $28.8 \pm 2.5 \mu \mathrm{m}$ (range: $23-38$ ) and $29.2 \pm 2.8$ $\mu \mathrm{m}$ (range: $22-35)$ respectively, $(\mathrm{p}=0.389)$. There was no significant correlation between of ganglionic cell-inner plexiform and macular retinal nerve fibre layer with daily dose $(p=0.229)$ or cumulative dose of hydroxychloroquine $(p=0.678)$.

Conclusion : The average thickness of ganglionic cell -inner plexiform cell layer was significantly lower in those taking hydroxychloroquine than in controls. Thinning of this layer could be an early indicator of retinal toxicity before the appearance of clinical retinopathy. However, thickness of the macular retinal nerve fibre laye showed no decrease in hydroxychloroquine users and did not correlate with the duration or cumulative doses of hydroxychloroquine. Therefore, macular retinal nerve fibre layer thickness is not a useful biomarker for the early detection of hydroxychloroquine retinal toxicity.

\section{Background}

Hydroxychloroquine (HCQ) is a well known treatment option for many rheumatological and autoimune disorders like systemic lupus erythematosus and rheumatoid arthritis. Howevere, many side effects were reported on various ocular parts like cornea, ciliary body, retina, and the choroid in the form of deposition , which are the classical form of ocular side effects, discontinuation of the medication may reverse most of the side effcts. However, Bulls eye maculopathy is an irreversible form of retinal toxicity that affects the macula and leads to loss of central visual field, loss of visual acuity, and color vision defects[1]. Animal studies confirmed that toxicity results in in damage to perifoveal retinal ganglionic cell.Early detection of toxicity before it is clinically visible on fundus examination is important, because immediate discontinuation of HCQ might reverse early retinal toxicity. Many investigations are conducted to detect early changes such as visual field test, ocular coherence tomography, and electroretinogram (ERG)[2]. 
HCQ induced retinal toxicity is a rare condition; but higher incidence has been reported in a recent study than it was previously known , and is dose dependent [1]. The toxcity rate can reach up to $1 \%$ if the total dose exceeds $1000 \mathrm{~g}$ and if used for more than 5 years,[2]. Furthermore, worsening of retinopathy may happen even after discontinuation of HCQ [1]. Risk factors for developing retinal toxicity are a daily HCQ dose of more than $400 \mathrm{mg}$, takind the drug more than 5 years, and a total exceeding $1000 \mathrm{~g}$ [3].Retinopathy can be detected subjectively via measuring the best corrected visual acuity , anterior segment assesment using slit lamp , examination of the retina after dilatation, and visual field 10-2 test [4]. Useful objective tests are : autoflourescent photos of the retina, multifocal ERG, and spectral domain OCT (SD-OCT) [5]. Screening recommendation is to perform a minimum of one of the three tests ( autoflourescent photo, ERG or OCT ) besides the subjective tests [6]. Retinal toxicity should be demonstrated on two screening tests, one being objective, before discontinuation of treatment in patients thought to have sighns of toxicity secondary to HCQ [7].

The purpose of this work was to investigate the thickess of ganglion cell inner plexiform layer (GC-IPL) and that of the macular retinal nerve fiber layer (RNFL) in patients on HCQ therapy, and to compare these values with those of age-matched controls. This might serve as a tool to detect early macular retinal changes before the changes are clinically visible.

\section{Methods}

The study was approaved by the the Institutional Review Board of the University of Jordan number $19 / 20 / 279$ and written informed consent was obtained from every participant in the study and it was performed in accordance with the Helsinki Declaration of 1964, and its later amendment.

This was a retrospective cross-sectional study. Data of patients on HCQ therapy who visited Jordan University Hospital between September 2018 and September 2019 and underwent a standard ophthalmic exam, including measurement of GL-IPL and macular RNFL by OCT was extracted. Thickness of GI-IPL and macular RNFL of age-matched healthy subjects was measured and compared. Patients on HCQ therapy and those in the control group underwent a complete ophthalmic examination including best corrected visual acuity, intraocular pressure measurement, dilated fundus exam, color examination using Ishihara pseudoisochromatic plates (Kanehara Shuppan Co. Ltd., Tokyo Japan) and OCT.

The inclusion criteria were: best visual acuity $\geq 0.8$, normal ophthalmic exam, intraocular pressure $<21$ $\mathrm{mmHg}$, normal appearance of the optic disk, and normal OCT macula and peri-papillary RNFL.

Exclusion criteria were as follows: history of ocular surgery, history of eye trauma, ocular inflammation, spherical equivalent more than 6 diopters, optic disc or retinal disease, or optic disc anomaly like glaucoma.

To measure macular thickness, an $8 \llbracket 8 \mathrm{~mm}$ macular area centered around the foveal thickness was recorded using the OPTOPOL SD OCT machine (SD OCT version 7.2.0, OPTOPOL Technology Sp. z.o.o., Poland). The central foveal and average macular thickness were recorded, with foveal thickness 
segmented by a single retinal specialist. The OCT machine differentiated between the retinal tissue interfaces and detected the thickness of GC-IPL. RNFL thickness (diameter $3.5 \mathrm{~mm}, 768$ A-scans) was also obtained. RNFL thickness from the inner margin of the internal limiting membrane to the outer margin of the RNFL layer was automatically segmented using OCT. A machine tracking system was used to compensate for any eye movement.

Statistical analysis:

We used SPSS version 21.0 (Chicago, USA) for analysis. Continuous variables (e.g., age) were described as mean ( \pm standard deviation). Other nominal variables (e.g., gender) were described as count (frequency). We performed an independent sample t-test to analyze the difference in the mean values between cases and controls. Paired-sample t-tests were used to analyze the mean differences in OCT measurements at baseline and at follow-up in both groups,using the filter function to isolate the study subjects and controls for the test. Data is presented as means ( $95 \%$ confidence interval [CI] ). Independent-sample t-tests were performed to analyze the mean difference in age between the groups and chi-square test was used to compare the frequency of gender and other risk factors between the groups. Pearson correlation coefficient was used to analyze the correlation between the OCT findings, duration of treatment, and cumulative dose of HCQ. The correlation between thickness of GL-IPL and macular RNFL, and daily and cumulative HCQ doses was analyzed. All underlying assumptions were met unless otherwise indicated. A p value of 0.05 was considered as statistically significant.

\section{Results}

In all, 219 patients were included in this study with a mean age of 43.38. \pm 17.39 . years (Table 1$)$. The study group comprised100 (20 male and 80 female) patients with a mean age of $45.28( \pm 12.24)$ years, and the control group comprised119 patients (44 males and 75 females), with a mean age of 41.79 ( \pm $20.67)$ years. There was no significant difference in age between the groups $(p=0.123)$. The mean cumulative dose of HCQ was $566.6 \pm 432.9 \mathrm{~g}$, and the mean duration of HCQ therapy was $56.5 \pm 36.3$ months. The mean daily dose was $313.9 \pm 96.9 \mathrm{mg}$. The participants' demographic data are presented in Table 1.

The average GC-IPL thickness was $85.9 \pm 8 \mu \mathrm{m}$ (Range: 50 - 98) in patients on HCQ therapy compared to $88.6 \pm 6.2 \mu \mathrm{m}$ (Range: $75-110$ ) in the control group. The average RNFL thickness was $29.2 \pm 2.8 \mu \mathrm{m}$ (range: 22 - 35) in patients on HCQ therapy and 28.8 $\pm 2.5 \mu \mathrm{m}$ (range: $23-38$ ) in ) in the control group.

There was a significant difference in the mean GCL-IPL thickness between the groups $(p=0.006)$, with a mean difference of $0.31 \mu \mathrm{m}$ (95\% confidence interval ranging from 0.79 to 4.56$)$; the mean thickness was higher in the control group. However, the average macular RNFL thickness was statistically similar in both groups $(p=0.389)$. Table 2 shows details of the differences between the groups.

There was no significant correlation between GCL+IPL and macular RNF thickness with either daily dose $(p=0.229)$ or the cumulative dose $(p=0.678)$, as demonstrated in Table 3. 
Table 4 shows the correlations between SD-OCT measurements (average RGC-IPL and RNFL thickness) and mean age of patients in both groups. There was a statistically significant negative relationship between age and average RGC-IPL thickness in patients on HCQ therapy $(p<0.05)$. There was no statistically significant correlation between the age of patients and average GC-IPL thickness in both groups $(p>0.05)$.

\section{Discussion}

Our study shows that the GC-IPL layer and macular RNFL thickness might serve as early diagnostic tools for detecting retinal toxicity due to HCQ use. However, the thickness was not negatively related to the duration and tolal intake of HCQ.

It was proposed that retinopathy secondary to HCQ alter early the ganglionic cells with possible secondary RNFL thinning and damage. Until recently, there are few studies with limitted ssample sizes, using variable imaging tecniques, different treatment regimens and short duration of follow up , moreover, the results are variable and conflicting [7] The exact way the HQC causes retinopathy is not well known. HCQ has high afinity to the the melanin in retinal pigment epitheluim and attaches to it and this may contribute to its toxic effects. Animal studies showed that accumilation of HCQ in the retinal ganglionic cells may affect the photoreceptors long before the RPE shows signs of toxicity [8]. It was demonstrated that SD-OCT photos demonstrated focal atrophy of retinal layers around the foveal area, and might indicate retinal toxicity long before occurrence of visual field loss $[7,8]$. Most previous studies investigated diseases affecting the retinal pigment epithelium and photoreceptors[9], other investigators have showed that HCQ can damage the inner part of the retina only in normal-looking retinae [7]; however, damage was observed in the inner and outer retina when abnormal fundus was clinically evident [10]. Few studies have evaluated the thickness of the GP-IPL layer; unfortunately, the number of subjects enrolled was small and the duration of HCQ therapy was short $[11,12]$ t. It was observed that RNFL thinning devolop after clinically visible HCQ retinopathy [13,14]. Moreover, inconsistent results were found regarding the relationship between GC-IPL thickness, and total dose and duration of HCQ therapy. While some invistigators demonstrated that dose and duration of HCQ correlate negatively with average GC-IPL thickness [11], another study demonstrated no significant association [7], which is consistent with our results. It was postulated that retinal ganglionic cells show changes as early as first week after starting treatment with HCQ therapy and in photoreceptors soon afterwards[8].This might explain our findings that changes in the GC-IPL were not related with the duration of treatment or cumulative dose of HCQ. However, paradoxical thickening of the macular RNFL was observed, compared to controls. The findings of this study are consistent with those reported in a similar study recently conducted by Lee et al. $[7,8]$. Thickening of the RNFL was observed in many ocular pathologies such as retinitis pigmentosa and drug-related changes. It was explained that it is a chronic reactive change, secondary to retinal ganglionic cell stroma and axonal degeneration $[15,16]$ The sample size in our study is larger than that in previous works, and the control group had no previous ocular factors that might affect the quality of the OCT scan. For each individual scan, each OCT was manually segmented to ensure good resolution. 
Our study has several limitations. First, is the retrospective nature and the small sample size, which was due to the low incidence of patients receiving HCQ therapy. Second, the control group consisted of healthy subjects. The ideal group would be an age-matched group with the same rheumatological disorders but not receiving HCQ therapy. This is difficult as it is not always possible to perform ocular examinations in patients with rheumatological disorders without any concrete reasons. Third, the crosssectional nature of the study made it difficult to evaluate the longitudinal effect of the medication. Further studies with long-term changes are required to confirm our findings and to investigate if thinning of the GC-IPL precedes retinopathy.

In conclusion, macular GC-IPL showed significant thinning in patients on HCQ therapy but did not correlate with the duration and cumulative dose. Therefore, GC-IPL and macular RNFL might serve as early biomarkers for HCQ toxicity.

\section{List Of Abbreviations}

OCT: ocular coherence toography

GC-IPL : ganglionic cell- inner plexiform cell layer

RNFL: retinal nerve fiber layer

ERG: and electroretinogram

SD-OCT: spectral domai ocular coherence tomography.

\section{Declarations}

Ethics approval and consent to participate: The study was approaved by the the Institutional Review Board of the University of Jordan number 19/20/279 and written informed consent was obtained from every participant in the study. The study was performed in accordance with the Helsinki Declaration of 1964 , and its later amendment

Consent to publish: all authors: all participants give the consent to publish the manuscript

Availability of Data and materials: The datasets generated during and/or analyzed during the current study are available from the corresponding author on reasonable request

Competing interests: All authers declare no conflict of interest

Funding: No funding or sponsorship was received for this study or publication of this article.

Authors' contributions: All named authors meet the International Committee of Medical Journal Editors (ICMJE) criteria for authorship for this article, take responsibility for the integrity of the work as a whole, and have given their approval for this version to be published. MS, AS, and MA have examined and 
collected the data , MS preformed the tomography, MS, AS,MA,JM, MQ,KE, and SR analyzed and interrupted the data, written the manuscript and did the leterature review. All authers had read and approved the final manuscript.

Acknowledgments: non aplicable

\section{References}

1. Kellner S, Weinitz S, Kellner U. Spectral domain optical coherence tomography detects early stages of chloroquine retinopathy similar to multifocal electroretinography, fundus autofluorescence and nearinfrared autofluorescence. Br J Ophthalmol 2009;93:1444-7.

2. Levy GD, Munz SJ, Paschal J, Cohen HB, Pince KJ, Peterson T. Incidence of hydroxychloroquine retinopathy in 1,207 patients in a large multicenter outpatient practice. Arthritis Rheum 1997;40:1482-6.

3. Wolfe F, Marmor MF. Rates and predictors of hydroxychloroquine retinal toxicity in patients with rheumatoid arthritis and systemic lupus erythematosus. Arthritis Care Res (Hoboken) 2010;62:77584.

4. Marmor MF, Kellner U, Lai TY, Lyons JS, Mieler WF. Revised recommendations on screening for chloroquine and hydroxychloroquine retinopathy. Ophthalmology 2011;118:415-22.

5. Costedoat-Chalumeau N, Ingster-Moati I, Leroux G, Fardeau C, Benveniste O, Simon C, et al. Critical review of the new recommendations on screening for hydroxychloroquine retinopathy. Rev Med Interne 2012;33:265-7.

6. Marmor MF. Efficient and effective screening for hydroxychloroquine toxicity. Am J Ophthalmol 2013;155:413-4.

7. Lee MG, Kim SJ, Ham DI, Kang SW, Kee C, Lee J, et al. Macular retinal ganglion cell-inner plexiform layer thickness in patients on hydroxychloroquine therapy. Invest Ophthalmol Vis Sci 2014;56:396402.

8. de Sisternes L, Hu J, Rubin DL, Marmor MF. Localization of damage in progressive hydroxychloroquine retinopathy on and off the drug: inner versus outer retina, parafovea versus peripheral fovea. Invest Ophthalmol Vis Sci 2015;56:3415-26.

9. Stepien KE, Han DP, Schell J, Godara P, Rha J, Carroll J. Spectral-domain optical coherence tomography and adaptive optics may detect hydroxychloroquine retinal toxicity before symptomatic vision loss. Trans Am Ophthalmol Soc 2009;107:28-33.

10. Chen E, Brown DM, Benz MS, Fish RH, Wong TP, Kim RY, et al. Spectral domain optical coherence tomography as an effective screening test for hydroxychloroquine retinopathy (the "flying saucer" sighn)clin Ophthalmol 2010;4:1151-8.

11. Bulut M, Erol MK, Toslak D, Akidan M, Basar EK and Cay HF. A New Objective Parameter in Hydroxychloroquine-induced Retinal Toxicity screening Test. Macular Retinal Ganglionic -Inner 
Plexiform layer Thickness. Arch Rheumatol 2018; 33(1):52-58 doi:

105606/ArchRheumatol.20180.6327

12. Xiaoyun MA, Dongyi HE, Linping HE. Assessing chloroquine toxicity in RA patients using retinal nerve fibre layer thickness, multifocal electroretinography and visual field test. $\mathrm{Br} \mathrm{J}$ Ophthalmol 2010;94:1632-6.

13. Bonanomi MT, Dantas NC, Medeiros FA. Retinal nerve fibre layer thickness measurements in patients using chloroquine. Clin Exp Ophthalmol 2006;34:130-6.

14. Pasadhika S, Fishman GA. Effects of chronic exposure to hydroxychloroquine or chloroquine on inner retinal structures. Eye (Lond) 2010;24:340-6.

15. Walia S, Fishman GA. Retinal nerve fiber layer analysis in RP patients using Fourier-domain OCT. Invest Ophthalmol Vis Sci 2008;49:3525-8.

16. Han J, Lee K, Rhiu S, et al. Linezolid-associated optic neuropathy in a patient with drug-resistant tuberculosis. J Neuroophthalmol 2013;33:316-8

\section{Tables}

Table 1

Description of the - overall characteristics of the study population

\begin{tabular}{|llllll|}
\hline & N & Minimum & Maximum & Mean & Std. Deviation \\
\hline Age & 219 & 9.0 & 91.0 & 43.384 & 17.3852 \\
\hline RNFL thickness(um) & 219 & 22.0 & 38.0 & 28.9 & 2.6 \\
\hline GCL + IPL thickness(um) & 219 & 50.0 & 110.0 & 87.37 & 7.2 \\
\hline Duration of HCQ therap (months) & 100 & 6.0 & 168.0 & 56.5 & 36.3 \\
\hline Accumulative dose in grams & 100 & 36000.0 & 2016000.0 & 566640.0 & 432896.6 \\
\hline Average daily dose in grams & 100 & 100.0 & 400.0 & 314.0 & 96.9 \\
\hline
\end{tabular}

Table 2

shows details of the differences between the groups.

\begin{tabular}{|c|c|c|c|c|}
\hline & Group & Mean thickness $(\mu \mathrm{m})$ & Std. Deviation & $p$ value \\
\hline \multirow[t]{2}{*}{ RNFL thickness } & HQC group & 29.160 & 2.8203 & \multirow[t]{2}{*}{0.389} \\
\hline & Control group & 28.849 & 2.5165 & \\
\hline \multirow[t]{2}{*}{ GCL + IPL thickness } & HCQ group & 85.910 & 7.9647 & \multirow[t]{2}{*}{0.006} \\
\hline & Control group & 88.588 & 6.1869 & \\
\hline
\end{tabular}


Table 3. Correlations between measured thicknesses and cumulative and daily HCQ dose, and duration of HCQ therapy

\begin{tabular}{|lllllll|}
\hline & \multicolumn{2}{l}{ Cumulative dose } & \multicolumn{2}{c|}{ Daily dose } & \multicolumn{2}{c|}{ Duration of therapy } \\
\hline & $\mathrm{R}$ & $\mathrm{p}^{*}$ & $\mathrm{R}$ & $\mathrm{p}^{*}$ & $\mathrm{R}$ & $\mathrm{p}^{*}$ \\
\hline $\begin{array}{l}\text { Avarage RGC- } \\
\text { IPL th }\end{array}$ & -0.042 & 0.678 & -0.121 & 0.229 & -0.046 & 0.649 \\
$\begin{array}{l}\text { Avarage } \\
\text { RNFL }\end{array}$ & -0.139 & 0.168 & -0.087 & 0.389 & -0.175 & 0.081 \\
$\begin{array}{l}\text { * Pearson's correlation test; RGC-IPL: Retinal ganglion cell-inner plexiform layer; RNFL: Retinal nerve } \\
\text { fiber layer }\end{array}$ & & & & & & \\
\hline
\end{tabular}

Table 4. Correlations between Age of both groups and Retinal ganglion cell-inner plexiform layer thickness and Retinal nerve fiber layer thickness.

\begin{tabular}{lllll|} 
& Group 1 & & Group 2 & \\
& $\mathrm{R}$ & $\mathrm{p}$ & $\mathrm{R}$ & $\mathrm{p}^{*}$ \\
$\begin{array}{l}\text { Average } \\
\text { GC-IPL }\end{array}$ & 0.000 & 0.678 & -0.241 & 0.008 \\
$\begin{array}{l}\text { Avarage } \\
\text { RNFL }\end{array}$ & 0.248 & 0.013 & & 0.453 \\
* Pearson's correlation test; RGC-IPL: Retinal ganglion cell-inner plexiform layer; RNFL: Retinal nerve \\
fiber layer
\end{tabular}

